\title{
Processamento Inicial de dados Magnetotelúricos na região da bacia sedimentar Tucano central: Análise Preliminar
}

\author{
Paulo Roberto Santos ${ }^{1}$, Andrea Santos-Matos ${ }^{12}$, Antonio Lopes Padilha ${ }^{2}$, Marcelo Banik Pádua ${ }^{2}$ \\ ${ }^{1}$ Universidade Federal do Pampa \\ ${ }^{2}$ Instituto Nacional de Pesquisas Espaciais - INPE/DIDGE/GEOMA - Grupo de Geomagnetismo
}

Copyright 2019, SBGf - Sociedade Brasileira de Geofísica

This paper was prepared for presentation during the $16^{\text {th }}$ International Congress of the Brazilian Geophysical Society held in Rio de Janeiro, Brazil, 19-22 August 2019.

Contents of this paper were reviewed by the Technical Committee of the $16^{\text {th }}$ International Congress of the Brazilian Geophysical Society and do not necessarily represent any position of the SBGf, its officers or members. Electronic reproduction or storage of any part of this paper for commercial purposes without the written consent of the Brazilian Geophysical Society is prohibited.

\begin{abstract}
In the present work we show the preliminary results from magnetotelluric (MT) data processing and the preliminary analysis of a linear profile deployed perpendicularly to the tectonic structures along the states of Bahia and Pernambuco, Brazil. To obtain an uniform distribution of the electrical conductivity of the crust and upper mantle in the SW portion of the Province Borborema, MT data were collected at 14 stations along a $240 \mathrm{~km}$ profile in the $\mathrm{E}-\mathrm{O}$ direction. The data was processed using robust techniques, which generated MT transfer functions. For the preliminary qualitative analysis of these responses, pseudo-sections were constructed with the interpolation of the phase and resistivity data into the two orthogonal directions of measurements ( $X Y$ and $Y X)$. Preliminary analysis suggests that the lithosphere under the São Francisco Craton (O portion of the profile) and the Tucano basin (E portion) are geoelectrically different.
\end{abstract}

\section{Introdução}

Este trabalho é parte do estudo que está sendo realizado com dados magnetotelúricos ao longo de um perfil, no nordeste do Brasil entre os estados da Bahia e Pernambuco. A região de estudo situa-se entre a província Borborema e o Cratón São Francisco (figura 1). Os dados estão localizados na região central da bacia sedimentar do Tucano e nos blocos Serrinha e Gavião. O método geofísico Magnetotelúrico (MT), é utilizado para determinar um modelo geoelétrico de subsuperfície terrestre dentro de um intervalo de frequências que variam de 0,0001 a $1000 \mathrm{~Hz}$ (Hertz).

Com objetivo de obter um modelo de distribuição de condutividade elétrica da crosta e manto superior na região de estudo, foram processados dados de 14 estações ao longo de um perfil com direção E-O, com extensão aproximada de $240 \mathrm{~km}$. Os dados foram processados utilizando técnicas robustas, gerando-se respostas das funções de transferência dos sinais MT. Para a análise qualitativa dessas respostas foram construídas pseudosseções a partir da interpolação das fases e resistividades nas duas direções ortogonais de medidas ( $X Y$ e $Y X)$. A análise preliminar sugere que dois
Terrenos geoeletricamente distintos (uma na região do Cráton do São Francisco e outro na região da bacia sedimentar do Tucano).

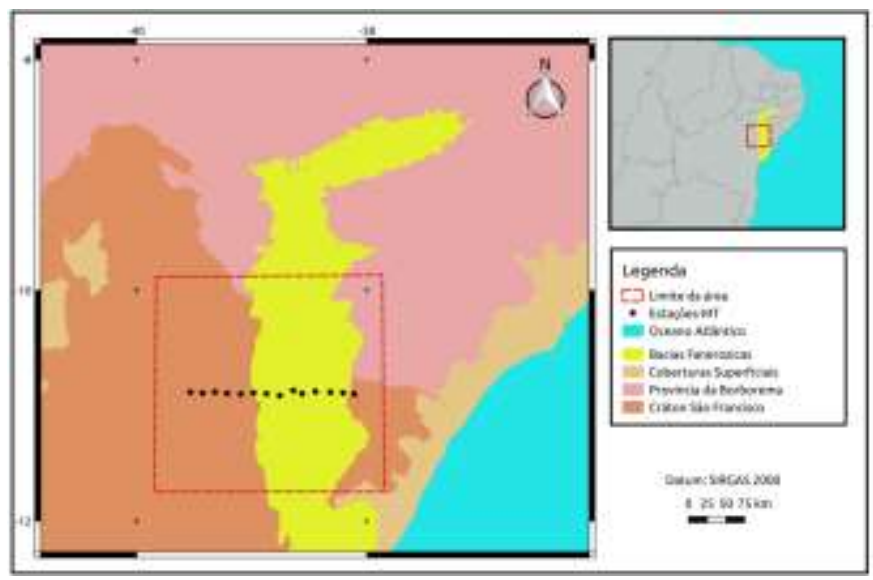

Figura 1. Mapa de localização das estações MT.

\section{Metodologia}

O método magnetotelúrico tem como objetivo imagear a distribuição de condutividade elétrica em subsuperfície, por meio de medidas da variação dos campos elétricos e magnéticos em superfície. A resistividade das litologias existentes é uma propriedade física específica de cada material. A variação dessa propriedade se deve a parâmetros como, porosidade da rocha, salinidade, temperatura e saturação. Esses parâmetros são fundamentais para caracterização de uma região (Jones, 1992).

Os dados foram processados seguindo procedimento adotado pelo grupo de pesquisa em Geomagnetismo da Divisão de Geofísica Espacial - GEOMA/DIDGE/INPE O processamento dos dados aqui realizados, chamado segundo Santos (2012) de pré-processamento, consiste de um conjunto de etapas que se inicia com a filtragem, tratamento estatísticos, conversão de arquivos, e transformação de domínios e agrupamento de arquivos. O fluxograma de pré-processamento apresentado na figura 2, ilustra as etapas do processamento dos dados magnetotelúricos. 


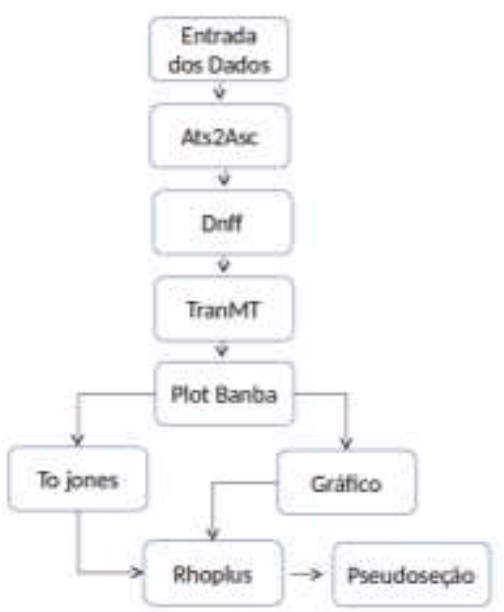

Figura 2. Fluxograma de pré-processamento de dados magnetotelúricos.

Depois de organizados, o primeiro passo consiste em converter dados binários ats originados do equipamento de aquisição .ats utilizando programa Ats2asc. A saída é um arquivo .txt. Após esse passo, os dados são passam por duas sub-rotinas: Dnff e TranMT. A primeira obtêm os coeficientes de Fourier das séries temporais por meio de análise espectral. (Egbert 1997). O TranMT é utilizado para fazer estatística robusta visando a retirada dos outliers para cada segmento. Posteriormente utiliza-se o programa ToJones para fazer a conversão do resultado da rotina anterior para que o próximo passo possa ser realizado. Uma vez convertido os dados, utiliza-se o programa Rhoplus (Parker e Booker, 1996), para verificar a consistência dos mesmos (respostas MT), antes de proceder com as etapas de modelagem ou inversão 1D ou 2D (Santos, 2012). No passo seguinte são geradas pseudosseção que consiste em uma primeira visualização dos dados MT.

\section{Resultados Preliminares}

As Figuras 3 e 4 apresentam as pseudossseções obtidas a partir da interpolação das fases nas direções ortogonais (xy e yx), resultado preliminar para o perfil estudado. Por não serem significativamente afetadas pelo deslocamento estático (static shift), as pseudosseções da fase permitem uma visão qualitativa inicial das principais estruturas geoelétricas da área analisada (Jones, 1988).

As Figuras 5 e 6 apresentam as pseudossseções obtidas a partir da interpolação das resistividades nas direções ortogonais ( $x y$ e $y x)$, resultado preliminar para o perfil estudado.

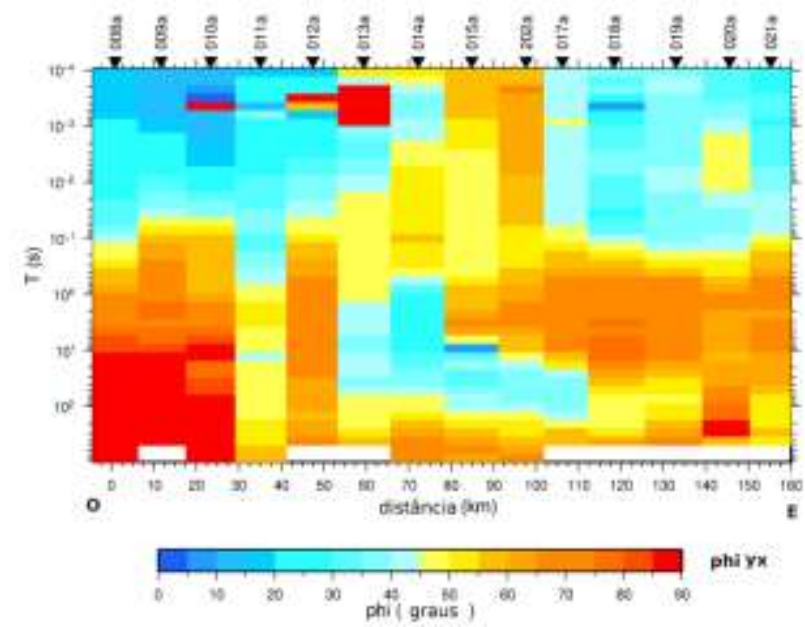

Figura 3. Peseudosseção da fase do perfil MT, componente $x y$.

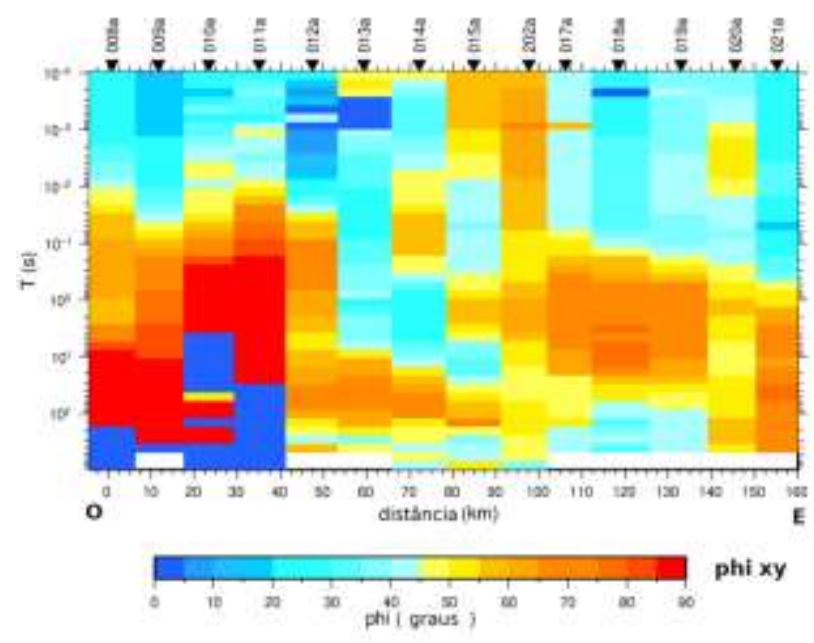

Figura 4. Peseudosseção da fase do perfil MT,

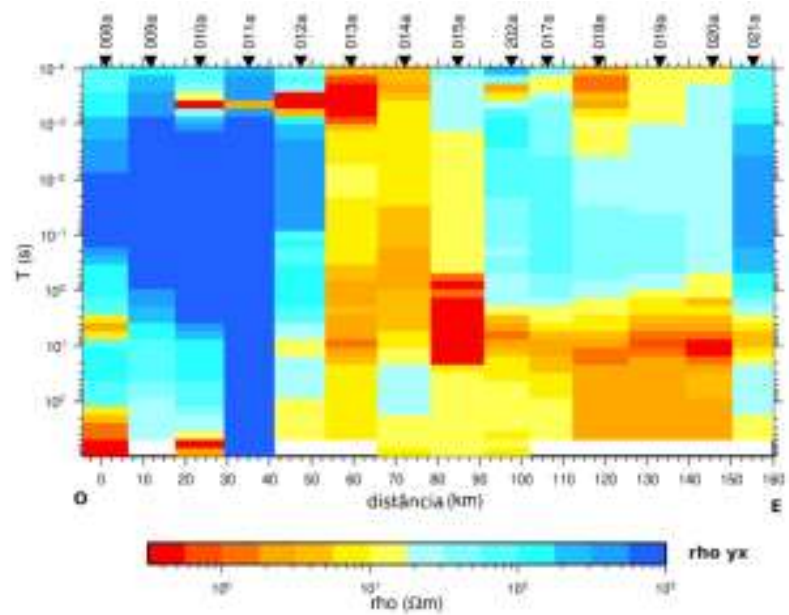

componente yx.

Figura 5. Peseudosseção de resistividade do perfil MT, componente yx. 


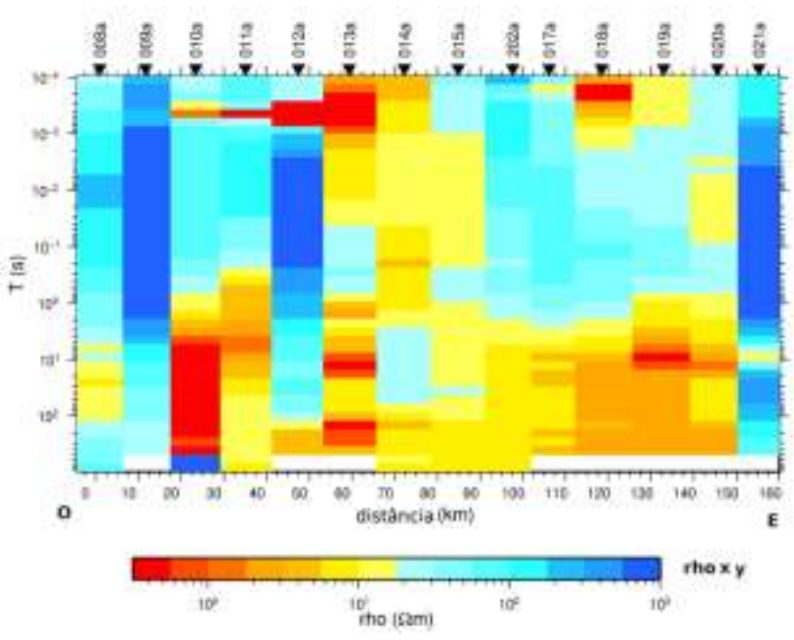

Figura 6. Peseudosseção de resistividade do perfil MT, componente $x y$.

As pseudosseções geradas a partir dos dados processados indicam, em geral, uma crosta superior resistiva com uma descontinuidade condutora na região da bacia sedimentar do Tucano central. Analisando as pseudosseções é possível observar presença de um condutor na crosta média ao longo de todo o perfil. $\mathrm{Na}$ crosta inferior nota-se uma estrutura geoelétrica distinta na porção $\mathrm{O}$ (que coincide com embasamento do cráton do São Francisco), mais resistiva que a estrutura observada a $\mathrm{E}$ (que coincide com o pacote sedimentar da bacia do Tucano).

\section{Conclusões}

A análise preliminar sugere que os Terrenos do Cráton do São Francisco (porção $O$ do perfil) e a região onde está localizada a bacia sedimentar do Tucano central (porção $E$ do perfil), são geoeletricamente distintas na crosta e manto superior. Após essa análise preliminar qualitativa dos dados, pretende-se em etapas posteriores realizar uma inversão 2D para definir melhor as estruturas geoelétricas identificadas nesse trabalho, corroborando com o conhecimento geotectônico local.

\section{Agradecimentos}

Os autores agradecem ao CNPq por disponibilizar bolsa de iniciação científica (PIBIC/INPE), que possibilitou a realização desse estudo.

\section{Referências}

Egbert, G.D. 1997. Robust multipleprocessing. Geophys. J. Int., 130, 475-496.s

Jones, A.G. Electrical conductivity of the continental lower crust. Continental lower crust, Elsevier, New York, p. 81143, 1992.
Jones, A.G. 1988. Static Shift of Magnetotelluric Data and its Removal in a Sedimentary Basin Environment. Geophysics, 53, 967-978.

Santos, A.C.L. 2012. Imageamento magnetotelúrico de estruturas dalitosfera na porção SE da província Borborema. Tese de Doutorado, Universidade de Brasília, 159 p. 